\title{
Effective Precoding Technique for Multiuser MIMO Systems*
}

\author{
Taoufik Ben Rejeb \\ Department of Information Security \\ Moscow Technical University of Communications and Informatics \\ Moscow, Russia \\ benrejebt@yandex.ru
}

\begin{abstract}
Using of quantized channel state information on transmitter (CSIT) side for adaptive signal processing on base station (BS) side also called as precoding provides to reduce feedback channel bandwidth in comparison with full CSIT. There are many solutions for feedback channel quantization but the aim of this paper is to note the performance of Grassmannian manifold for codebook design.
\end{abstract}

Keywords: Multiuser MIMO, MU-MIMO, CLTD-OSIC precoding, Grassmann manifold, quantizaion codebook.

\section{INTRODUCTION}

The current radio communication standards include the spatial processing of signals implying the use of MIMO (Multiple-Input-Multiple-Output) multi-antenna systems [1]. The use of MIMO technology in communication systems makes it possible to significantly increase the capacity, but involves the necessity of solving many problems of signal processing. The task of adaptive signal processing for the simultaneous operation for several users on the same allocated frequency-time resources falls on the computing power of both the BS and users sides.

Precoding performed on the BS side based on the CSIT obtained with feedback channel allows to calculate the necessary weights for the most efficient transmission of information over the communication channel.

\section{SYSTEM MODEL}

We consider a multiuser MU-MIMO system with spatial division multiple access (SDMA) [1], which consist of multiple antenna BS and several users where each of them has multiple antenna receivers. Let's introduce the following notations: $N$ - number of antennas on the BS side; $M-$ sum number of antennas on the user side; $k$ - index number of user $(k=1,2, \ldots, K) ; K$ - total number of users in a multiuser system; $M_{k}$ - number of antennas on $k$-th user side $\mathbf{H}_{k}$ complex channel matrix of dimension $M \times N$, where each element of $\mathbf{H}_{k}$ is a complex channel multiplier between user antennas and $\mathrm{BS}$ antennas; $\mathbf{H}=\left[\begin{array}{llll}\mathbf{H}_{1}^{T} & \mathbf{H}_{2}^{T} & \ldots & \mathbf{H}_{K}^{T}\end{array}\right]$ general matrix of MIMO channel between all users and BS of dimension $K \times N$, which consists of channel matrices $\mathbf{H}_{k} ; \mathbf{T}_{k}$ precoding matrix $r$ dimension of $M_{k} \times N$; $\mathbf{T}=\left[\begin{array}{llll}\mathbf{T}_{1} & \mathbf{T}_{2} & \ldots & \mathbf{T}_{K}\end{array}\right]$ - general precoding matrix of BS dimension of $N \times K$, which consist of precoding matrices $\mathbf{T}_{k}$ for $K$ users, where each of them contains the weighting factors for signals transmitted be BS. The structure of general BS's precoding matrix consisting of precoding vectors is shown in Figure 1.

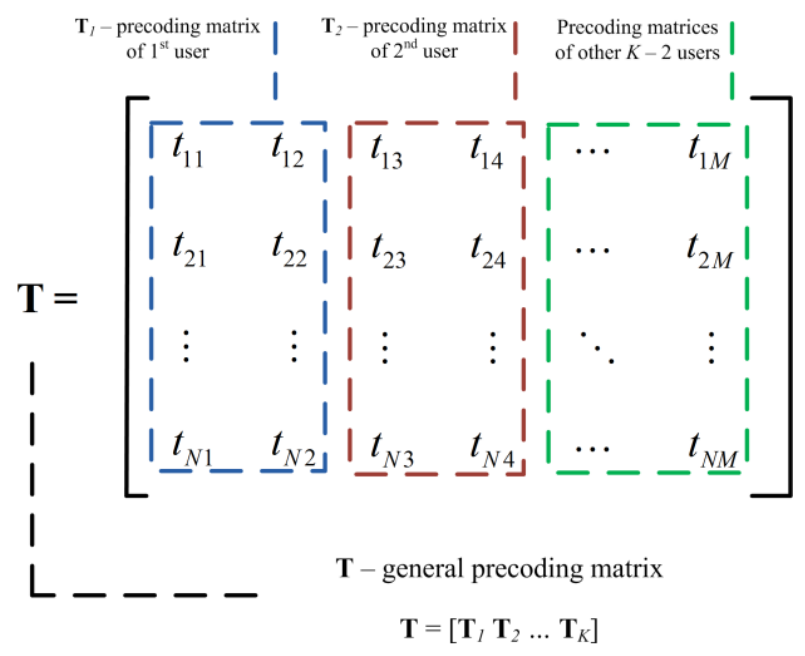

Fig. 1. Structure of general BS's precoding matrix

The main task of a base station is simultaneous data transmission for all $K$ users using $N$ transmit antennas and the same time-frequency resources. The precoding procedure is performed on the BS side, where the weights for the transmitting signals for each user are given by the common precoding matrix $\mathrm{T}$. The calculation of the matrix $\mathrm{T}$ is based on CSIT obtained by feedback channel from the each user to the $\mathrm{BS}$. 
The signal observed at the $k$-th receiver can be represented as follows [1]:

$$
\mathbf{y}_{k}=\mathbf{H}_{k} \mathbf{T}_{k} \mathbf{s}_{k}+\sum_{j=1, j \neq k}^{K} \mathbf{H}_{k} \mathbf{T}_{j} \mathbf{s}_{j}+\mathbf{n}_{k},
$$

where $\mathbf{y}_{k}$ - vector of received symbols observed at the $k$-th receiver; $\mathbf{s}_{k}$ - symbol vector which was transmitted from BS to $k$-th user; $\mathbf{T} j$ - precoding matrix of other $j$-th user, where $j$ $\neq k ; \mathbf{n}_{k}$ represents the vector of additive noise at the $k$-th receiver. Second part of (1) represents interfering signals of other users of the system with respect to the signal of the $k$-th user.

The precoding procedure requires the feedback channel having sufficient bandwidth for periodically transmitting CSIT to BS's side. The formation of such a feedback channel may require resources (time slots or frequency bands) that are maximally used for data transmission channels.

Since the presence of feedback channels is necessary for using the precoding procedure, the actual task is to find ways to reduce the amount of resources (temporary or frequency) needed to organize such channels.

\section{LIMITED FEEDBACK}

Precoding based on partial channel information was used in known standards of wireless communication systems such as LTE and LTE-Advanced, where we can find term "codebook-based precoding" [4]. The use of such precoding technique requires a codebook, known on the BS and AT side. The codebook is a set of vectors (codewords) consisting of complex channel multipliers. With a known codebook user compares the channel estimate with each of the codebook vectors, and transmits through the feedback channel to the BS only the index number of the selected vector. Quantization of the channel state information allows the transmission of only certain index numbers through the feedback channel represented by $B$ bits, which determine the result of the calculation of the precoding vectors on the BS side. The number of bits $B$ depends of the codebook $\mathrm{F}$ used on the user side for quantizing the channel state information. For transmission of quantized channel information to the BS side, the CDI (Channel Direction Information) indicator [6] is used. For estimation of signal level on the user side, the CQI (channel quality information) indicators are used. To evaluate the signal power, user terminals use the SINR - Signal Interference + Noise Ratio [4].

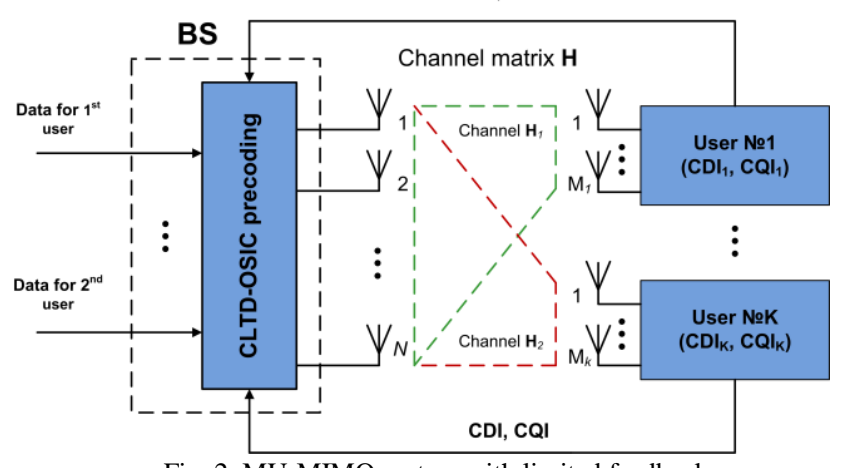

Fig. 2. MU-MIMO system with limited feedback

\section{CODEBOOK DESIGN AND SELECTION CRITERIA}

User's codebook can be represented as an $L \times N$ matrix F consisting of $L / M_{k}$ matrices dimension of $M_{k} \times N$ :

$$
\mathbf{F}=\left[\begin{array}{llll}
\mathbf{F}_{1} & \mathbf{F}_{2} & \ldots & \mathbf{F}_{L}
\end{array}\right], L=2^{B},
$$

where $L=2^{B} ; l-$ the index number of the codeword. An illustration of user's codebook structure is shown on Figure 3.

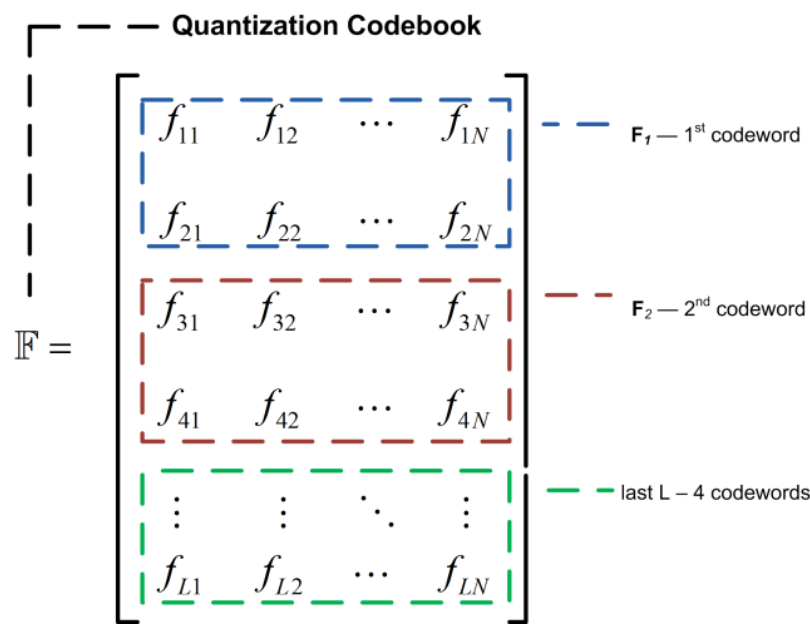

Fig. 3. User's codebook structure

The subscriber terminal codebook is an $L \times N$ dimension matrix, where each row matrix of this codebook is a $L \times N$ codeword. The codewords are used by subscriber terminals as quantized values of channel state information (Fig. 4). The size of the user's codebook depends of the number of bits $B$ transmitted on the feedback channel from the user to BS. High-dimensional codebook requires a larger value of $B$, and therefore a wider feedback channel, which is not always possible.

Availability of quantized channel vector at base station side makes it possible to calculate or select the precoding matrix. There are two approaches to feedback quantization:

- error calculation between the channel state information and codeword and deciding of precoding matrix on the BS side [10];

- $\quad$ selection the precoding vector on the user side by using different criteria [10].

In the case of the first approach, the following difference criterion is applicable:

$$
\mathbf{F}_{l}=\arg \max _{\mathbf{F}_{l} \in \mathrm{F}}\left\|\mathbf{H}_{k} \tilde{\mathbf{F}}_{l}\right\|_{F}
$$

Authors in [9] propose to generate codebooks of users based on Grassmann manifold. Grassmannian $\mathbb{G}_{K, N}$ or Grassmann manifold is the set of subspaces of dimension $K$ in $N$-dimensional complex space. Using of Grassmann manifold packing provide to geometrically represent codewords of codebook $\mathrm{F}$ as matrices of various dimensions in a given multidimensional space. Using of Grassmannian package make it possible to arrange codewords represented by subspaces in a multidimensional space using various metrics.

There are different distance metrics between subspaces [9], but for the case of user's equipment with 
multiple antenna, the codebook will consist of Grassmanian subspaces (matrices). In this case, the distance metric is applicable:

- Fubini-Study metric:

$d_{F S}\left(\mathbf{F}_{l}, \mathbf{F}_{m}\right)=\arccos \left|\operatorname{det}\left(\mathbf{F}_{m}^{H} \mathbf{F}_{l}\right)\right|$,

- Chordal distance metric:

$d_{c h}\left(\mathbf{F}_{l}, \mathbf{F}_{m}\right)=\frac{1}{\sqrt{2}}\left\|\mathbf{F}_{l} \mathbf{F}_{l}^{H}-\mathbf{F}_{m} \mathbf{F}_{m}^{H}\right\|_{F}$

\section{SIMULATION RESULTS}

The results of computer simulation, presented in Table, allow to compare the results of noise immunity of MUMIMO systems with proposed CLTD-OSIC precoding technique using grassmannian manifold and MMSE precoding based on full channel matrix knowledge.

\section{SIMULATION PARAMETERS}

\begin{tabular}{|l|l|}
\hline Channel & $\begin{array}{l}\text { MIMO rayleight fading } \\
\text { channel }\end{array}$ \\
\hline Antenna configuration & $4 \times 2 \times 2$ \\
\hline $\begin{array}{l}\text { Number of antennas on BS } \\
\text { side }\end{array}$ & 4 \\
\hline $\begin{array}{l}\text { Number of antennas on user } \\
\text { side }\end{array}$ & 2 \\
\hline Number of users & 2 \\
\hline Transceiver architecture & V-BLAST \\
\hline Channel encoding & $\begin{array}{l}\text { Convolution encoding } \\
\text { (rate 1/2) }\end{array}$ \\
\hline Precoding technique & CLTD-OSIC \\
\hline Number of feedback bits & 6 bits \\
\hline Demodulator & MMSE \\
\hline Modulation & QPSK \\
\hline Number of experiments & 100000 \\
\hline
\end{tabular}

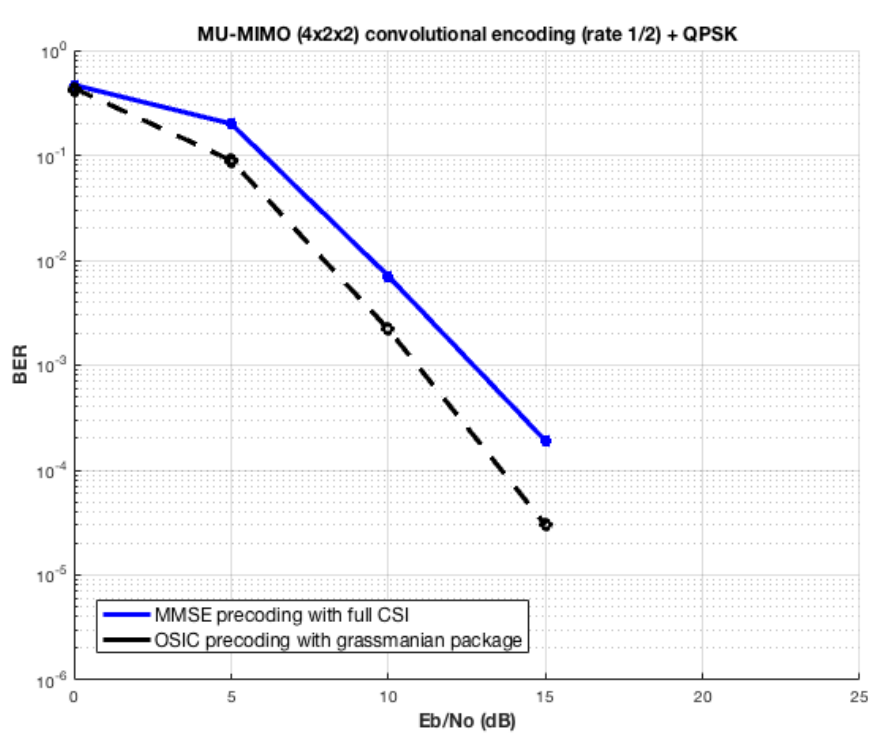

Fig. 4. Simulation results

As shown on Figure 4, using of proposed CLTD-OSIC precoding technique based on Grassmannian manifold provides to gain $2 \mathrm{~dB}$ of the energy efficiency in comparison with known MMSE precoding technique based on full channel matrix knowledge.

\section{REFERENCES}

[1] Бакулин М.Г., Варукина Л.А., Крейнделин В.Б. Технология MIMO: принципы и алгоритмы. М.: Горячая линия - Телеком, 2014. [Bakulin M.G., Varukina L.A., Kreyndelin V.B. MIMO Technology: Principles and Algorithms. Moscow: Hotline - Telecom, 2014].

[2] Kreyndelin V., Smirnov A., Ben Rejeb T. Effective precoding and demodulation techniques for $5 \mathrm{G}$ communication systems // Proc. Systems of Signals Generating and Processing in the Field of on Board Communications. 2018. P. 1-6.

[3] Brown T., De Carvalho E., Kyritsi P. Practical guide to the MIMO radio channel: with MATLAB examples. Chichester: John \& Wiley Sons Ltd, 2012.

[4] Sibille A., Oestges C., Zanella A. MIMO: From Theory to Implementation. : Elsevier Ltd., 2011.

[5] Yoo T., Jindal N., Goldsmith A. Multi-Antenna Broadcast Channels with Limited Feedback and User Selection // IEEE Journal Sel. Areas in Communications. 2007. P. 1478-1491.

[6] Santipach W., Honig M. Asymptotic capacity of beamforming with limited feedback // Proc. IEEE Int. Symp. Inform. Theory (ISIT). 2004. July. P. 290.

[7] Brown III D.R., Love D.J. MIMO Nullforming with RVQ Limited Feedback and Channel Estimation Errors // Proceedings of the 48th Asilomar Conference on Signals, Systems, and Computers. Pacific Grove, CA, November 2-5, 2014. Pacific Grove, 2014.

[8] Yoo T., Jindal N., Goldsmith A. Multi-Antenna Broadcast Channels with Limited Feedback and User Selection // IEEE Journal on Selected Areas in Communications. 2007. Vol. 25, N 7.

[9] Medra A., Davidson T. Flexible codebook design for limited feedback downlink systems via smooth optimization on the Grassmannian manifold // IEEE 13th International Workshop on Signal Processing Advances in Wireless Communications. 2012.

[10] Tsoulos G. MIMO System Technology for Wireless Communications. Boca Raton: CRC Press, 2006. 